\title{
Smartphone: en comunicación, algo más que una adicción
}

\section{Smartphone: in communication more than addiction}

Ubaldo Cuesta Cambra. Universidad Complutense de Madrid. España. ucuestac@ucm.es

$[\mathrm{CV}]$ (1)

Victoria Cuesta Díaz. Hospital Quirón Madrid. España.

victoria.cuesta.diaz@gmail.com

$[\underline{\mathrm{CV}}]$

Luz Martínez Martínez. Universidad Complutense de Madrid. España.

luzmar03@ucm.es

$[\underline{\mathrm{CV}}]$ (1) $\mathrm{G}$

José Ignacio Niño González. Universidad Complutense de Madrid. España.

jnino@ucm.es

$[\mathrm{CV}](\mathrm{C}$

Cómo citar este artículo / Referencia normalizada

Cuesta Cambra, U., Cuesta Díaz, V., Martínez Martínez, L. y Niño González, J. I. (2020).

Smartphone: en comunicación, algo más que una adicción. Revista Latina de Comunicación

Social, 75, 367-381. https://www.doi.org/10.4185/RLCS-2020-1431

\section{RESUMEN}

Diferentes trabajos han analizado la adicción al Smartphone (Young, 2009; Beard, 2005). Sin embargo, todavía no existe una base teórica sólida que permita interpretar desde la psicología social este trastorno adictivo, ni una herramienta de diagnóstico precoz breve derivada de este fundamento (Cuesta, Cuesta y Martínez, 2019). Se hace indispensable la creación de herramientas que permitan estudiar los mecanismos psicosociales que subyacen al uso problemático del Smartphone, y que aporte conocimientos que permitan analizar sus fundamentos psicosociales y posibles perfiles de uso. Objetivo: La creación de un cuestionario breve de adicción/uso problemático al Smartphone que permita investigar los diferentes perfiles psicosociales de uso. Método: Un meta análisis de la bibliografía permitió detectar los factores psicosociales validados empíricamente sobre los cuales existe mayor consenso. Posteriormente se realizaron tres grupos de discusión y cinco entrevistas en profundidad entre expertos y profesionales. Con estos datos se elaboró un cuestionario abreviado que fue cumplimentado por una muestra de jóvenes universitarios en dos ocasiones (test-retest). Resultados: El cuestionario obtuvo adecuados valores de Cronbach y test-retest en todos los ítems. El análisis factorial realizado permitió encontrar una estructura factorial de 4 factores de uso que fueron denominados: envolventes, socializados, virtualizados y estresados. Estas denominaciones, que remiten a los factores psicosociales presentes con mayor peso en cada factor, parecen indicar la existencia de diferentes patrones de uso problemático del móvil lo cual supone replantearse la adicción al Smartphone no como un único concepto y admitir la existencia de diferentes tipos de adicción. En un futuro se deberá explorar la existencia de factores y su vinculación a patrones de personalidad. 
PALABRAS CLAVE: adicción comportamental, comunicación salud, escala adicción Smartphone; patrones consumo.

\begin{abstract}
Different research have analysed Smartphone addiction (Young, 2009; Beard, 2005). However, there is still no solid theoretical basis that allows interpreting this addictive disorder from social psychology, nor a tool for a brief early diagnosis derived from this foundation (Cuesta, Cuesta \& Martínez, 2019). It is essential to create tools to investigate the psychosocial mechanisms that underlie the problematic use of the Smartphone, and that provide knowledge that understand its psychosocial foundations and usage profiles. Objective: The goal has been the creation of a brief questionnaire of addiction/problematic use to the Smartphone and to investigate the different psychosocial profiles of use. Method: A meta-analysis of the literature allowed us to detect the empirically validated psychosocial factors on which there is greater consensus. Subsequently, three discussion groups and five in-depth interviews between experts and professionals were conducted. With these data, an abbreviated questionnaire was prepared and completed by a sample of university students twice (test-retest). Results: The questionnaire obtained adequate Cronbach and test-retest values in all the items. The factor analysis made it possible to find 4 structural factors that were called: enveloping, socialized, virtualized and stressed. These denominations, which refer to the psychosocial factors present with greater weight in each factor, seem to indicate the existence of different patterns of problematic use of the mobile which means rethinking the addiction to the Smartphone not as a single concept and admitting the existence of different types of addiction. In the future, the existence of factors and their link to personality patterns should be explored.
\end{abstract}

KEYWORDS: behavioural addiction; health communication; personality addiction; Smartphone addiction scale.

\title{
CONTENIDO
}

1. Introducción. 2. Objetivo. 3. Método. 4. Resultados. 5. Discusión y limitaciones. 6. Referencias bibliográficas.

Traducción de Carlos Javier Rivas Quintero (Universidad de los Andes, Mérida, Venezuela).

\section{Introducción}

El Smartphone se ha convertido en un objeto indispensable para el día a día, gracias a su capacidad para conectarse a Internet de forma fácil y cada vez más barata, por su capacidad para hacer fotos y videos, así como por la posibilidad de acceso a numerosas plataformas y aplicaciones bien para comunicarse, buscar o compartir información. Todo ello lo convierten en una herramienta de conexión entre sujetos o de mero entrenamiento indispensable en la vida de los individuos y entre los jóvenes (Herrera-Batista, 2009). Según el informe del Estudios General de Medios (EGM) a principios de 2019 , un tercio $(31,2 \%)$ de los consumidores diarios de Internet en España son adolescentes y jóvenes de 14 a 34 años (EGM, 2019) cuyo principal dispositivo de acceso es el Smartphone $(95,6 \%)$ haciendo uso sobretodo de la mensajería instantánea $(95,8 \%)$, redes sociales $(61,3 \%)$, uso de aplicaciones $(53,4 \%)$ y mantenerse informado de la actualidad (44,5\%). En cuanto al uso de redes sociales, este rango de edad representa el 39\% de la población. De media, los españoles pasan 2,27 horas al día conectados al móvil mientras que el $49 \%$ de los jóvenes entre 18 y 24 pasan más de 4 horas (Ditrendia, 2019). Sin embargo, lo recomendado, para un uso considerado noperjudicial, es de 2 horas al día como máximo (OECD, 2015). Además, su uso comienza cada vez antes, como muestra la encuesta sobre Equipamiento y Uso de Tecnologías de Información y 
Comunicación en los Hogares del Instituto Nacional de Estadística (2019): el 66\% de la población de 10 a 15 años dispone de teléfono móvil y el 90\% a partir de los 13 años.

En el momento en que el uso del Smartphone impacta negativamente en el desarrollo de la vida cotidiana social, laboral, estudiantil o familiar se puede definir como "uso problemático del móvil" (Acier \& Kern, 2011; Billieux, Maurage, López-Fernández, Kuss \& Griffiths, 2015) cuyas consecuencias pueden ser: pérdida de tiempo, alteraciones de la conducta, de los ritmos del sueño, falta de control, aislamiento, bajo rendimiento, etc. (Park \& Rang, 2014; Vilca \& Vallejos, 2015). Los jóvenes continúan usando el Smartphone a pesar de que les genera dificultades sociales o problemas de tipo interpersonal (Odriozola, Labrador \& Becoña, 2009) ya que el uso recurrente y constante de esta conducta puede hacer que no cumplan con sus obligaciones en el trabajo, la escuela o el hogar. En este contexto, se apunta el papel de padres, especialistas y educadores en la educación y control de un uso útil y no problemático del móvil (Godoy, 2017).

Por todo ello, el estudio del uso excesivo o problemático del móvil y su influencia en la conducta, generan gran interés, por la naturaleza adictiva y sus implicaciones sobre la salud pública.

Diferentes trabajos han analizan la adicción sin sustancias y el uso abusivo de conductas relacionadas con las nuevas tecnologías, como al juego, a Internet o a los videojuegos (Young, 2009; Beard, 2005; Fisher, 1994). Estos trabajos se han centrado en medir el uso, su grado e intensidad de los diferentes servicios. Muy pocos trabajos han analizado, hasta el momento, los motivos, desde el punto de vista pisco-social, del uso excesivo del dispositivo en sí. El DSM-5 (López, Honrubia \& Freixa, 2012; Cuesta, Cuesta \& Martínez, 2019) clasifica la adicción en función de variables como la tolerancia, la abstinencia, el deseo o intención por evitar la conducta, el tiempo dedicado, la disminución en otras actividades y la incapacidad para dejar de realizar una actividad nociva (American Psychiatric Association, et al., 2014), pero sin entrar en consideraciones de tipo psico-social sobre sus posibles causas.

Otras investigaciones sobre el uso del Smartphone muestran que genera en las personas diferentes sensaciones relacionadas con la independencia, el estatus, la seguridad, el control y el ocio (GarcíaRuiz, Ruiz \& Gómez, 2018), pero también hay ciertas conductas negativas relacionadas con Internet y las redes sociales que afectan a la salud y bienestar de los usuario, tales como el phubbing, el cual provoca que el usuario sea indiferente al entorno o a las personas que le rodean, prestando su máxima atención al dispositivo móvil (Urbina, 2014) provocando problemas en las relaciones interpersonales y laborales (Treviño, 2013), la nomofobia o miedo a estar sin el móvil, trastornos del ritmo del sueño, que hace que uno de cada cuatro jóvenes revise notificaciones de sus redes sociales en mitad de la noche (Ditrendia, 2018) o el síndrome de FoMO (fear of missing out), el "miedo a sentirse fuera", a perder el contacto y de las actividades que organizan los demás (Kuss \& Griffiths, 2011). En su investigación sobre el uso problemático del móvil con jóvenes de 12 y 19 años, Santana, Gómez \& Feliciano (2019) comprobaron una correlación entre una mayor frecuencia de uso con un mayor nivel de FoMO, además de una menor comunicación con los padres.

Se hace indispensable la creación de herramientas que permitan estudiar estos mecanismos psicosociales que subyacen al uso del Smartphone, especialmente cuando se produce un uso excesivo $\mathrm{o}$ adictivo, y que aporte conocimientos para entender sus bases psicológicas. Además, considerando que el objetivo principal de una herramienta de estas características consiste en la realización de screenings en entornos reales (colegios, fundamentalmente), es necesario que el cuestionario sea "muy breve" con objeto de permitir una aplicación rápida, eficaz y sencilla. 


\section{Objetivos}

La presente investigación tiene como objetivos: 1) conocer las motivaciones y contextos de uso del Smartphone de los jóvenes 2) la creación de un cuestionario breve de adicción al Smartphone para su diagnóstico precoz o screening, 3) analizar su fiabilidad y validez interna 4) investigar la existencia de diferentes patrones psicosociales de uso. Para ello se plantean las siguientes preguntas de investigación:

RQ1: ¿Cuáles son las motivaciones conductuales de jóvenes hacia el uso del Smartphone y problemas derivados de ellos?

RQ2: ¿Cuáles serán los ítems más adecuados para configurar un cuestionario muy breve que incluya los aspectos psicológicos, sociales y comportamentales que pueden subyacer a la conducta de adicción al Smartphone, así como sus índices de fiabilidad?

RQ3: ¿Permitirá el análisis de la validez interna del cuestionario encontrar patrones o clusters de conducta que reflejen diferentes estilos de uso del Smartphone?

\section{Método}

Para identificar, desde una perspectiva psico-social, los ítems del cuestionario se llevó a cabo una revisión de la bibliográfica existente sobre las escalas que se encuentren validadas y que midan el uso del Smartphone en los buscadores especializados Pubmed, Scopus y en el repositorio de revistas digitales de la Universidad Complutense de Madrid con los valores "mobile pone" "cell-phone", "communication", "behavior", "addictive”, "personality", "Smartphone”, "scale” y "questionnaire". Se obtuvo una muestra final de 27 trabajos que fueron codificados según la escala de evaluación de adicción utilizada, factores de evaluación, finalidad del cuestionario, resultados y nivel de significación de la escala. Un análisis de los ítems empleados por estas escalas permitió revisar los principales "componentes" usados en la literatura académica para definir esta conducta y empleados con mayor frecuencia en las escalas de evaluación.

Para estudiar la psicología de los jóvenes y de sus padres y disponer de datos para profundizar en los motivos de la conducta, con el objetivo de construir la escala, se realizaron tres reuniones de grupo (focus group) de 10 componentes cada uno: GD1 con adolescentes estudiantes de secundaria (14-16 años), GD2 con jóvenes universitarios (18-20 años) y GD3 con padres de adolescentes usuarios de Smartphones.

Con el mismo objetivo, se realizaron cinco entrevistas en profundidad con expertos en: (E1) drogadicción, comportamientos sociales y nuevas adicciones sin sustancias, (E2) Internet y comunidades virtuales, (E3) creación y difusión de plataformas de redes sociales utilizadas principalmente por adolescentes, (E4) joven usuario experto en video-juegos y (E5) responsable de comunicación social.

Durante los grupos de discusión y las entrevistas en profundidad, un miembro del grupo de investigación se encargó de que se cumplieran los requisitos necesarios para la fluidez del discurso alrededor de los objetivos establecidos en la investigación. Las sesiones fueron grabadas y posteriormente transcritas para un análisis de los datos que permitiera: 1) la extracción de los literales o verbatims que conformaran las unidades básicas de estudio y 2) conocer la opinión de expertos acerca de la potencialidad adictiva del Smartphone, la situación actual del uso entre los jóvenes, sintomatología, etc. 
Basándonos en el análisis bibliográfico y resultados de los focus group y de las entrevistas en profundidad, se elaboró el cuestionario de 9 ítems en escala Likert de 7 puntos, siendo 1= No (nunca) y $7=$ siempre.

Posteriormente el cuestionario fue cumplimentado por una muestra de 66 sujetos jóvenes universitarios, con el objeto de evaluar su fiabilidad y su validez interna.

La fiabilidad se analizó mediante la prueba Alfa de Cronbach que permitió calcular el coeficiente de fiabilidad en cuanto a su consistencia interna. También se analizó su fiabilidad mediante la prueba test-retest: el cuestionario fue cumplimentado por los mismos sujetos una semana después, lo que permitió calcular los índices de correlación de los 9 ítems que conforman la escala, obteniendo así los valores de fiabilidad en cuanto a la estabilidad de la escala.

Finalmente, mediante una prueba de análisis factorial, se analizó la validez de constructo llamada validez estructural o factorial. El análisis de esta validez estructural nos permitió analizar los diferentes tipos de uso del Smartphone al interpretar los clusters en función de los ítems del cuestionario que saturaban en cada factor.

\section{Resultados}

RQ1: ¿Cuáles son las motivaciones conductuales de jóvenes hacia el uso del Smartphone y problemas derivados de ellos?

Para conocer los aspectos motivacionales de los jóvenes hacia el uso del Smartphone se analizaron los datos obtenidos en adolescentes, jóvenes, padres y especialistas en el campo mediante los focus group y entrevistas en profundidad realizadas.

Las respuestas y comentarios fueron agrupadas en seis grandes categorías: razones por las que se conectan, hábitos de conexión, edad, papel de los padres, papel de los educadores, adicción o dependencia y futuro. Estas seis categorías fueron divididas a su vez en 14 subcategorías (tabla 1).

Tabla 1. Categorización de la información tras las entrevistas individuales y grupales.

\begin{tabular}{|l|l|}
\hline CATEGORÍAS & SUBCATEGORÍAS \\
\hline 1. ¿Por qué se conectan? & 1.1 ¿Cuál es el "gancho" de los Smartphones? \\
& 1.2 Accesibilidad y disponibilidad \\
& 1.3 Ventajas frente a desventajas \\
\hline 2. Hábitos de conexión & 2.1 ¿Cuántas veces al día? ¿Cuánto tiempo? \\
& 2.2 ¿Dónde? ¿Cuándo? \\
& 2.3. ¿Cantidad versus calidad? \\
\hline 3. Edad & \\
\hline 4. Papel de los padres & 4.1. Conocimientos tecnológicos de los padres \\
& 4.2. Control paterno/relación padres-hijos \\
& 4.3. Actualización tecnológica de las aptitudes paternas \\
& 4.4.Educación familiar \\
\hline 5. Papel de los educadores & 4.5. Hábitos y patrones familiares \\
\hline 6. ¿Momento de hablar de adicción o & 5.1. Actualización proyecto curricular \\
dependencia? & 6.1. Control-Madurez \\
\hline
\end{tabular}

Fuente: elaboración propia. 
En relación con las razones de por qué los jóvenes se conectan, los sujetos coinciden en que el gancho del Smartphone es su utilidad para facilitar la comunicación y socialización ya que "puedes estar en contacto permanentemente" (GD3), con herramientas como "WhatsApp" (GD2) y "estar informado de lo que pasa en el mundo" (E1). Tanto los jóvenes como profesionales son conscientes de que su fácil accesibilidad y disponibilidad facilitan su uso y convierten al Smartphone en una herramienta presente en la mayoría de los jóvenes, que suelen disponer de varios dispositivos "tengo uno (ordenador) en mi habitación, otro en el salón, y luego tablets y Play (...), para no aburrirnos" (GD1) y cada vez con mejores servicios "cada vez se nos ofrecen mejores tablets, móviles más polivalentes, gadgets más baratos y esto hace que la gente quiera tener el último móvil”. (E2). Aunque se reconozca su utilidad para la comunicación, los informantes dan un mayor peso a sus posibles desventajas o consecuencias no deseables, donde la esfera de lo privado pasa a ser público resultando excesivo "es como el show de Truman, todo el mundo sabe todo" (E2) y creando un condicionamiento social de compartirlo todo "es que te llega (una foto) y tú no tienes la opción de no aceptarla" (E1).

Sobre los hábitos de conexión, todos los sujetos declararon estar constantemente conectados, "lo uso todos los días, bueno, de lunes a viernes un poco menos porque estudio y luego ya hasta que me duermo con el móvil en la mano" (GD1), "uso el móvil todos los días principalmente para el WhatsApp y luego llamar" (E4); sin ser conscientes de que esto suponga algún tipo de problema "mis padres me dicen que no pase tanto tiempo con el móvil, (...) no sé," (E5). Sin embargo, los jóvenes utilizan el móvil antes, durante y después de hacer las tareas, antes de irse a la cama, al despertarse, en la mesa, en la ducha, si salen con los amigos e incluso en clase, aunque esté prohibido "todos días a todas horas, en todas las clases... es que es así" (E5), "en clase hay algunos que son adictos al Instagram, están todo el día conectados" (GD1). Aunque los jóvenes y adolescentes declaraban el uso del móvil como herramienta de comunicación y socialización, el aumento de la cantidad de tiempo que pasan conectados no es sinónimo de aumento de la calidad de ese proceso socializador "puedes tener 700 amigos en Facebook pero en realidad te hablas con 10 y tienes bloqueados a los demás para no ver sus fotos". (E2), "a lo mejor tienes ahí a gente que no es amigo tuyo" (GD2).

Todos los informantes coinciden en señalar que la edad de inicio de los jóvenes en la utilización del Smartphone es cada vez menor "se empieza antes, (...) antes a los 17 años y ahora a los 13" (P2.GD3), "mi hijo de 4 años maneja el iPhone que no veas". (P3.GD3); propiciado por los padres como modo de poder localizar a los niños "el teléfono se lo tenemos que dejar por si le pasa algo y tenerla localizada" (P2. GD3), "si tiene una hora de transporte público y ves que se retrasa pues coges y la llamas para quedarte tranquila" (P4.GD3). Aunque hay una edad límite para poder abrirte una cuenta en una red social, los adolescentes reconocen que mienten para poder registrarse "mucha gente miente respecto a la edad, al hacer el registro en una red social, para poder registrarse; saltándose por ejemplo la restricción de Tuenti de los 14 años” (EP1).

En cuanto al papel de los padres, existe en todos los grupos una posición discursiva predominante de que los conocimientos que tienen los padres con respecto al manejo de las nuevas tecnologías, en general, y los móviles de última generación, en particular, están muy por debajo de la de sus hijos "los padres tienen menos control tecnológico que los hijos. Los hijos están más adelantados." (E2), "por desgracia yo no sé hacer un seguimiento de eso, porque no sé. Sé wasapear y no sé nada más" (M3.GD3). Además, y quizás como algo derivado o consecuencia de ello, se identifica igualmente, que el nivel de utilidad que le sacan los padres a estas herramientas también es menor que el que obtienen los jóvenes "nos quejamos, pero es lo que hay. Hay cosas que no las controlo y me dice: mamá te estás quedando anticuada” (M1.GD3). En el caso del control paterno, los padres realizan distintas acciones para controlar el tiempo de consumo y saber el tipo de información que consumen 
sus hijos y quiénes son sus amistades virtuales tales como establecer horarios "a nosotros nos han puesto un horario, entre semana 15 minutos después de la tarea" (GD1), "los fines de semana somos más permisivos" (GD3); o comprobar el historial y sitios visitados "es casi obligado que como padre hay que ver dónde se han metido (en favoritos, en qué páginas)” (E1). Sin embargo, tanto expertos como jóvenes sugieren como más conveniente una posición pro-tecnológica por parte de los padres que fomente la confianza de sus hijos "compartir una cuenta de correo electrónico entre el padre y el hijo protege frente a un uso inadecuado." (E3), "pero vamos, tampoco tengo mucho problema. Yo tengo Facebook y a mi madre la agregué y todo y no me importa" (GD2). Los sujetos dieron mucha importancia a la educación familiar para la adquisición de madurez y control en un uso responsable por parte de los jóvenes “(...) Importancia de este colectivo (los padres) a la hora de educar, orientar y limitar a sus hijos en el uso del Smartphone" (E2), "hay que enseñarles a que sean responsables. Los padres tenemos que estar también un poquito encima de ellos, mirando" (GD3), "decirles que hay un límite y que ellos mismos se pongan ese límite, aquí empieza tu libertad y aqui tienes que saber dónde acaba" (GD2); sin embargo, se identificaron ciertos factores que pueden entorpecer este programa educacional como el problema de conciliación laboral y familiar ("en este país la conciliación laboral y la familiar no casan. A mí me gustaría levantarme a las 5 o a las 6 para tener las tardes libres y poder pasarla con mis hijos" (GD3)) o las "malas" influencias de ciertos contextos familiares que predisponen a que los adolescentes hagan un uso inadecuado ("lo mio es peor, mi hija vive con el WhatsApp y con el Tuenti y el Instagram desde que se levanta, pero es que a su padre le pasa lo mismo. Le regaña a ella, pero él es peor”(GD3)).

Sobre el papel de los educadores, los sujetos coincidieron en la necesidad de cambiar el modelo educativo tradicional con formación y reflexión sobre el uso adecuado del móvil entre los jóvenes y fijar criterios de diferenciación entre la información útil y segura y la no válida ("debe de haber una tutela, una vigilancia, probablemente una formación (...) ni siquiera sé (...), porque en los aspectos cibernéticos evidentemente hay más riesgos que los del mero abuso. Desde esa perspectiva yo creo que sí procede hacer una formación especifica" (E3).

Es importante saber hasta qué punto controlan los jóvenes el uso de su móvil y si demuestran suficiente madurez a la hora de utilizarlo, la mayoría de los jóvenes se declararon maduros y controlando su uso, así como la percepción de los especialistas ("la inmensa mayoría hace un uso responsable y saludable de estas herramientas" (E1); aunque se identificaron ciertos síntomas generalizados como la pérdida de horas de sueño, olvidos, nerviosismo ante la idea de no poder usarlo o la necesidad de utilizarlo como distracción ("que alguien procese en su cabeza a las 3 de la mañana subir al Tuenti o al Facebook una foto... que se ponga a etiquetar es duro" (E2) ;"como se rompa el móvil de un hijo la que se arma en casa es brutal. No pueden vivir sin él” (GD3).

RQ2: ¿Cuáles serán los ítems más adecuados para configurar un cuestionario muy breve que incluya los aspectos psicológicos, sociales y comportamentales que pueden subyacer a la conducta de adicción al Smartphone, así como sus índices de fiabilidad?

Los focus groups, junto con las entrevistas en profundidad arrojaron unas conclusiones que permitieron comprender la psicodinámica de la adicción al Smartphone.

Empleando estos resultados, y teniendo en consideración los trabajos previos analizados en la revisión bibliográfica, se diseñó el cuestionario breve de adicción al Smartphone de nueve ítems que se presenta a continuación: 
Tabla 2. Propuesta de cuestionario de adicción al Smartphone.

\begin{tabular}{|c|c|c|c|c|c|c|c|}
\hline Pseudónimo & $\begin{array}{l}\text { J્巳 } \\
\text { 莺 } \\
\text { Z }\end{array}$ & & & & & & 竞 \\
\hline En una escala que va del 1 al 7 & 1 & 2 & 3 & 4 & 5 & 6 & 7 \\
\hline "Duermo con él y puede emitir avisos por la noche" & & & & & & & \\
\hline $\begin{array}{l}\text { "Me gusta responder inmediatamente llamadas, } \\
\text { mensajes, mails...incluso si estoy ocupado/a en otra } \\
\text { cosa" }\end{array}$ & & & & & & & \\
\hline $\begin{array}{l}\text { "Me ha molestado que alguna vez me critiquen por } \\
\text { usarlo mucho" }\end{array}$ & & & & & & & \\
\hline $\begin{array}{l}\text { "Ojalá no me quitara tanto tiempo porque podría } \\
\text { hacer más cosas" }\end{array}$ & & & & & & & \\
\hline $\begin{array}{l}\text { "A veces lo uso un poco a escondidas para que no } \\
\text { me riñan" }\end{array}$ & & & & & & & \\
\hline "Sentiría mucha angustia si me lo quitaran" & & & & & & & \\
\hline $\begin{array}{l}\text { "Siento como una necesidad interna de consultarlo } \\
\text { con frecuencia (ver correos, ver mis redes } \\
\text { sociales..." }\end{array}$ & & & & & & & \\
\hline $\begin{array}{l}\text { "Me gusta mucho que me llamen o me escriban o } \\
\text { aparezca en las redes sociales de mis amigos" }\end{array}$ & & & & & & & \\
\hline $\begin{array}{l}\text { "Me inquieta la posibilidad de que digan algo malo } \\
\text { de mí en las redes sociales" }\end{array}$ & & & & & & & \\
\hline
\end{tabular}

Fuente: elaboración propia.

El análisis descriptivo de los ítems obtenidos en la primera ejecución, mostró los valores más altos para los ítems "siento la necesidad de consultarlo con frecuencia" (valor medio=4,74), "sentiría mucha angustia si me lo quitaran" (media=4,58) y "me gusta responder de forma inmediata" $(4,30)$. Por el contrario, los valores más bajos aparecieron en los ítems "a veces lo uso un poco a escondidas" $(2,08)$, "me ha molestado que me critiquen por usarlo mucho" $(2,47)$ y "me inquieta la posibilidad de que digan algo malo de mí en las redes" $(2,52)$.

La fiabilidad del cuestionario fue explorada mediante el análisis de fiabilidad de Cronbach y la prueba de test-retest.

Los resultados de Cronbach son elevados $(0,61 ; p<, 000)$, especialmente para una escala breve, dado que el valor Alfa de Cronbach se incrementa habitualmente con el tamaño de la muestra. Este resultado indica que los diferentes ítems del cuestionario manifiestan valores estables y que miden de forma fiable aquello que pretenden medir, sin que se produzcan diferencias significativas aleatorias por el mero paso del tiempo (tabla 3).

Tabla 3. ANOVA con Test de Cronbach.

\begin{tabular}{|l|l|l|l|l|l|l|}
\hline \multicolumn{2}{|l|}{} & Sum of Squares & Dfach & Mean Square & Cochran's Q & Sig \\
\hline Between People & 387,382 & 63 & 6,149 & & \\
\hline \multirow{3}{*}{ Within People } & Between Ítems & 546,406 & 8 & 68,301 & 159,357 &, 000 \\
\cline { 2 - 7 } & Residual & 1209,149 & 504 & 2,399 & & \\
\cline { 2 - 7 } & Total & 1755,556 & 512 & 3,429 & & \\
\hline Total & 2142,938 & 575 & 3,727 & & \\
\hline
\end{tabular}

Fuente: elaboración propia. 
Para evaluar la prueba test-retest, se realizó un análisis de correlaciones de Pearson entre los resultados del cuestionario y de la repetición del mismo para cada uno de los nueve ítems. Todos los ítems arrojaron correlaciones significativas $(\mathrm{p}<.000)$.

Los resultados de las correlaciones son los que se muestran a continuación, para cada uno de los ítems:

ITEM 1: ,72

ITEM 2: ,49

ITEM 3:, 70

ITEM 4: ,73

ITEM 5:, 44

ITEM 6: ,71

ITEM 7: ,81

ITEM 8: ,50

ITEM 9: ,71

Finalmente, con el objetivo de analizar la estructura interna del cuestionario y de los posibles perfiles psicológicos de los usuarios, se realizó un análisis factorial (tabla 2) que ayude a proponer un modelo psicológico de adicción al Smartphone.

Tabla 4: Matriz de componentes de análisis factorial.

\begin{tabular}{|l|c|c|c|c|}
\hline \multirow{2}{*}{} & \multicolumn{4}{|c|}{ Componente } \\
\cline { 2 - 5 } & $\mathbf{1}$ & $\mathbf{2}$ & $\mathbf{3}$ & $\mathbf{4}$ \\
\hline Dormir con él &, 007 &, 062 &, 740 &, 016 \\
\hline Responde inmediatamente &, 549 &, 213 &, 163 &,- 398 \\
Molesta críticas &, 444 &,- 567 &,- 088 &,- 051 \\
\hline Ojalá hacer más uso &, 748 &,- 296 &,- 150 &, 215 \\
\hline Usar a escondidas &, 680 &,- 469 &,- 003 &,- 059 \\
\hline Angustia sin él &, 534 &, 250 &, 353 &, 126 \\
\hline Necesidad consultar &, 665 &, 300 &, 206 &,- 247 \\
\hline Aparecer en redes sociales &, 282 &, 784 &,- 003 &, 245 \\
Miedo mala reputación &, 342 &, 222 &,- 243 &, 745 \\
\hline Edad &,- 406 &,- 065 &, 414 &, 230 \\
\hline Sexo &, 034 &, 539 &,- 452 &,- 386 \\
\hline
\end{tabular}

Fuente: elaboración propia.

El análisis muestra cuatro factores que explican en total el 61,15\% de la varianza:

Factor 1, al que hemos denominado "Uso envolvente". Se trata de sujetos "envueltos" en la actividad constante del móvil, el cual probablemente se utiliza con mucha frecuencia y para todo tipo de actividades. Parece saturar en todos los ítems del cuestionario, excepto en la necesidad de dormir con él, lo cual parece dar un índice de que se trata de una conducta controlable, no tan impulsiva como para necesitar dormir con él y permitir que despierte al usuario por la noche con avisos.

Factor 2, denominado "Socializadores". En este factor, el mayor interés parece ser "estar conectados a las redes, estar presentes". Parece indicar que se trata de sujetos cuyo principal interés consiste en 
socializar, estar presentes en las redes y también conocer qué pasa en las redes. Es interesante que son usuarios que saturan negativamente en el ítem "recibir críticas por usarlo" y "usarlo a escondidas": esto podría indicar que su necesidad de socializar se amplía también al mundo real dado que no reciben críticas ni lo usan a escondidas, en contra de lo que le ocurría al grupo de sujetos del factor 1 (envolvente). Futuros estudios podrían incluir variables de personalidad o valores que permitieran medir la "tendencia a socializar" o, quizá también la "tendencia ser líder", con el objetivo de analizar las correlaciones con este factor.

Factor 3, denominado "Virtualizados". Aquí, el móvil, en cierto sentido, "forma parte de su cuerpo" y no pueden prescindir de él en ningún momento, aunque sin altos niveles de ansiedad. Saturan fundamentalmente en los ítems "dormir con él" y "sentirían angustia de perderlo". Son sujetos que parecen estar fuertemente motivados para emplear constantemente el móvil o, al menos, de sentir su presencia física a su lado (dormir con él, sentir angustia si lo pierdo...) pero que no manifiestan ansiedad por las críticas en las redes o por otros motivos.

En un sentido similar al anterior, futuras investigaciones deberían incluir, al evaluar rasgos de personalidad, evaluar el rasgo ansiedad o similar, con objeto de investigar las posibles interacciones con esta tipología.

Factor 4, al que hemos 1lamado "Estresados". Este actor satura positivamente en "el miedo a la mala reputación" y negativamente en "responder de forma inmediata". Así no parecería tanto una conducta compulsiva cuanto una especie de fuerte "preocupación" por el ciberespacio y las redes, lo que les lleva a estar más en contacto con ellas, pero sin el componente lúdico de los sujetos que hemos denominado "Socializadores".

Estos resultados parecen indicar, como habíamos hipotetizado, que existen diferentes "patrones" de uso del móvil, en función de las motivaciones psico-sociales que subyacen en su empleo y a las consecuencias percibidas: los usuarios de "uso envolvente", quizá los menos propensos a desarrollar conducta adictiva o problemática, los usuarios "socializadores", con alta motivación social y deseo de interacción con sus iguales, los "virtualizados", altamente involucrados en las redes sociales, pero sin mostrar ansiedad y, finalmente, los "estresados", quizá los sujetos con propensión a desarrollar conductas más patológicas con el uso del Smartphone.

Por lo tanto, más que de altos porcentajes de uso como concepto global, es quizá necesario comenzar a especificar qué tipo de uso hace la persona del móvil, dado que existen, al menos, cuatro patrones claramente diferenciados de empleo.

\section{Discusión y limitaciones}

La percepción del Smartphone es la de una herramienta atractiva que facilita la socialización y la comunicación, aunque se reconocen ciertos inconvenientes como la falta de privacidad, cambios en las formas de relación social, aislamiento y deterioro en las relaciones sociales (Urbina, 2014; Treviño, 2013). Los jóvenes son conscientes de que su accesibilidad y disponibilidad ha facilitado que lo utilicen de forma diaria, que lo utilizan incluso en entornos donde su uso está prohibido y reconocen su nerviosismo ante la idea de no poder usarlo, actitud reconocida también por los padres, sin embargo, no tienen la percepción de que esto suponga un problema, quedando normalizado. Por eso es importante tanto el papel de los padres como de los educadores para que los jóvenes hagan un uso saludable del móvil, aunque existen ciertos retos al mostrar una brecha respecto al conocimiento y estrategias de uso entre estos y los jóvenes, tal y como han mostrado nuestros resultados en los grupos de discusión y las entrevistas en profundidad. 
Todo ello es, por otro lado, coherente con datos recientes publicados al respecto en trabajos similares (Observatorio Nacional de Telecomunicaciones y de la Sociedad de la Información, 2019; INE, 2019). Esto sugiere la necesidad de diseñar nuevas estrategias de comunicación que formen, no sólo a los jóvenes, sino a los padres y educadores en un uso útil y responsable de estos dispositivos.

El cuestionario sobre el uso del Smartphone elaborado en esta investigación, obtuvo adecuados índices de fiabilidad tanto de consistencia interna (índice Alfa Cronbach) como de estabilidad (correlaciones de Pearson). Su validez de constructo, estructural o factorial (realizada mediante análisis factorial) parece muy adecuada y proporciona tipologías psicosociales de gran interés, tanto para el adecuado diagnóstico rápido tipo screening, como para el diseño posterior de programas de intervención. Investigaciones anteriores citan porcentajes globales elevados de uso del móvil (Herrera-Batista, 2009; INE, 2019) sin embargo, nuestros datos muestran una tasa variable de utilización en función de diferentes "patrones", siendo necesario especificar el tipo de uso que hace la persona, por lo que se podría hablar de diferentes tipos de consumidores, seguramente con diferentes patrones de adicción o de uso intensivo. Algunos de ellos, incluso aunque hicieran un uso muy intensivo de él, seguramente no podría hablarse, en sentido estricto, de adicción sino de patrones de consumo problemático del móvil, dado que no parecen generar ansiedad ni vincularse a otras variables propias de la adicción.

Así, podemos hablar de jóvenes que lo utilizan constantemente, pero con cierto control sobre su uso, los que lo utilizan para socializar, los que no podrían prescindir de él a nivel funcional y los preocupados por lo que opinan de él y sus acciones en Internet y en las redes sociales.

Futuras investigaciones con muestras mayores podrán ayudar a profundizar en posibles diferencias entre estos factores, especialmente si se emplean de forma complementaria medidas de factores de personalidad como la extroversión, la estabilidad emocional y las medidas de valores sociales, como la búsqueda de liderazgo o de socializar. Esto permitiría comprender mejor las bases psicológicas de la adicción al Smartphone permitiendo, no sólo definir los posibles patrones de uso, sino también correlacionarlos con determinados perfiles psicológicos, lo cual fortalecería el cuestionario como herramienta de detección precoz del uso problemático y abriría nuevos espacios a diferentes tipos de tratamientos. De este modo, al poder detectar determinados perfiles asociados a patrones de posible uso problemático se podrían diseñar estrategias útiles y eficaces para la educación y prevención. En este sentido, es también necesario investigar las diferencias de género y sus interacciones con los factores encontrados.

También será necesario investigar la correlación entre los elevados índices de uso del Smartphone y variables de validez externa concurrente, como el rendimiento académico. Es muy posible que los diferentes patrones discriminen unos mejor que otros las repercusiones sobre conductas como el rendimiento escolar, la ansiedad, el aislamiento, etc.

\section{Referencias bibliográficas}

Acier, D. \& Kern, L. (2011). Problematic Internet use: perceptions of addiction counsellors. Computers \& Education, 56(4), 983-989. https://doi.org/10.1016/j.compedu.2010.11.016

American Psychiatric Association, Kupfer, D. J., Regier, D. A., Arango, C., Ayuso-Mateos, J. L., Vieta, E. y Bagney, A. (2014). DSM-5: Manual diagnóstico y estadístico de los trastornos mentales. Editorial Médica Panamericana. 
Beard, K. W. (2005). Internet Addiction: A Review of Current Assessment Techniques and Potential Assessment Questions. CyberPsychology \& Behavior, 8(1), 7-14. http://doi.org/10.1089/cpb.2005.8.7

Billieux, J., Maurage, P., López-Fernandez, O., Kuss, D. J. \& Griffiths, M. D. (2015). Can disordered mobile phone use be considered a behavioral addiction? An update on current evidence and a comprehensive model for future research. Current Addiction Reports, 2(2), 156-162. https://doi.org/10.1007/s40429-015-0054-y

Cuesta, U., Cuesta, V. y Martínez, L. (2019). Adicción al Smartphone. Análisis descriptivo de herramientas de medición del uso del teléfono inteligente. En S. Morales Calvo, F. Vidal Auladell \& M. Mut Camacho (Coords.), Nuevo paradigma comunicativo: Lo 2.0, 3.0 y 4.0 (pp. 157-170). GEDISA.

Ditrendia, 2018. En Mobile en España y en el Mundo 2018. https://cutt.ly/BrGKezY

Estudio General de Medios (2019). Audiencia de Internet (abril 2018-marzo 2019), AIMC. https://www.aimc.es/a1mc-c0nt3nt/uploads/2019/04/internet119.pdf

Fisher, S. (1994). Identifying video game addiction in children and adolescents. Addictive behaviors 19(5), 545-553. https://doi.org/10.1016/0306-4603(94)90010-8

García, R., García, R. y Hernando, A. (2018). Redes sociales y estudiantes: motivos de uso y gratificaciones. Evidencias para el aprendizaje. Aula abierta, 47(3), 291-298. https://doi.org/10.17811/rifie.47.3.2018.291-298

Godoy, L. F. (2017). Fotografías en el aula: ¿Nuevas prácticas de lectura y escritura mediadas por TIC? Centre d'Estudis Vall de Segó; Quaderns Digitals, 85, 108-125.

Herrera, M. (2009). Disponibilidad, uso y apropiación de las tecnologías por estudiantes universitarios en México: perspectivas para una incorporación innovadora. Revista Iberoamericana de Educación, 48(6), 1-9. https://doi.org/https://doi.org/10.35362/rie4862130

Instituto Nacional de Estadística (2019). Encuesta sobre Equipamiento y Uso de Tecnologías de Información y Comunicación en los Hogares. Año 2018. https://www.ine.es/prensa/tich 2019.pdf

Kuss, D. J. \& Griffiths M.D. (2011). Online social networking and addiction - a review of the psychological literature. International Journal of Environmental Research and Public Health, 8(9), 3528-3552. https://doi.org/10.3390/ijerph8093528

López, O., Honrubia, M. L. y Freixa, M. (2012). Adaptación española del "Mobile Phone Problem Use Scale" para población adolescente. Adicciones, 24(2), 123-130. https://doi.org/10.20882/adicciones.104

Martínez, E., Martínez, J. y Martínez, M. A. (2019). Análisis del uso de dispositivos móviles en las aulas universitarias españolas". Revista Latina de Comunicación Social, 74, 997-1013. https://doi.org/10.4185/RLCS-2019-1368

Observatorio Nacional de Telecomunicaciones y de la Sociedad de la Información (2019). La Sociedad en Red. Transformación digital en España. Informe Anual 2018. 
https://www.ontsi.red.es/ontsi/sites/ontsi/files/201910/InformeAnualLaSociedadEnRedEdic2019.pdf

OECD (2015). Students, Computers and Learning. Making the Connection. OECD Publishing.

Odriozola, E., Labrador, F. J. \& Becoña, E. (2009). Adicción a las nuevas tecnologías en adolescentes y jóvenes. Pirámide.

Park, C. \& Rang, Y. (2014). The conceptual model on smart phone addiction among early childhood. International Journal of Social Science and Humanity, 4(2), 147-150. https://doi.org/10.7763/IJSSH.2014.V4.336

Santana, L. E., Gómez, A. M. y Feliciano, L. (2019). Uso problemático del móvil, fobia a sentirse excluido y comunicación familiar de los adolescentes. Comunicar: Revista científica iberoamericana de comunicación y educación, 59, 39-47. https://doi.org/10.3916/C59-2019-04

Treviño, J. (2013). El 'phubbing', otra amenaza para las relaciones interpersonales y laborales. Abogados.

Urbina, K. (2014). Impacto del phubbing en las relaciones. Tuxpan.

Vilca, L. \& Vallejos, M. (2015). Construction of the risk of addiction to social networks scale. Computers in Human Behaviour, 48, 190-198. https://doi.org/10.1016/j.chb.2015.01.049

Young, K. (2009). Internet Addiction: Diagnosis and Treatment Considerations. Journal of Contemporary Psychotherapy, 39(4), 241-249. https://doi.org/10.1007/s10879-009-9120-x

\section{AUTORES:}

\section{Ubaldo Cuesta Cambra}

Departamento de Comunicación Audiovisual y Publicidad II. Universidad Complutense de Madrid. España.

Catedrático de Comunicación Audiovisual y Publicidad de la UCM. Director del Departamento de Comunicación Audiovisual y Publicidad II y de la Cátedra de Comunicación y Salud de la UCM. Dr. En Psicología. Director del Máster de Publicidad UCM-agencia JWT y del Neurolabcenter. Director del Departamento de Teorías y Análisis de la Comunicación. Director de la revista Comunicación y Salud y de la revista Pensar la Publicidad (PLP) Director del grupo de investigación consolidado UCM "Creación y efectos psicosociales y culturales de la comunicación". Investigador principal de varios proyectos MINECO en el pasado. Actualmente I.P. del proyecto MINECO sobre “Antivacunas en la Red". Director de la Cátedra de Comunicación y Salud UCM-Abbvie. Exdirector de Publicidad de Citroën. Director del Laboratorio de Neurocomunicación de la UCM (Neurolabcenter.com). Director del Máster propio de Publicidad "Gestión Publicitaria" UCMWunderman-Thompson. Director del Máster propio de Comunicación y Salud UCM-Ayuntamiento de Madrid-Madrid Salud. Exdirector del Máster propio de Gestión de Televisión UCM-Telemadrid. Exdirector del Experto Creatividad y Planificación Estratégica UCM-Grey-Expiga. Vocal del Consejo Español de Drogadicciones y otras Adicciones. Autor de tres libros especializados en Psicología de la Comunicación y de siete libros como coordinador. Autor de más de 30 artículos en revistas de investigación de impacto en español e inglés (incluyendo varios Scopus y JCR), en el ámbito de la Psicología y la Comunicación. Sus líneas de investigación se centran en el estudio de 
diferentes aspectos de la Comunicación y Salud como adicciones, prevención y promoción de la salud. Análisis y creación de estrategias en Publicidad y Salud. Innovación de desarrollo en neurociencia, su aplicación en el neuromarketing y publicidad y el estudio de los fundamentos neuropsicológicos de la Comunicación.

ucuestac@ucm.es

Índice H: 8

Orcid ID: https://orcid.org/0000-0001-7023-7132

Google Scholar: https://scholar.google.com/citations?user=ysHqNCsAAAAJ\&hl=en

ResearcherID: H-5730-2015

\section{Victoria Cuesta Díaz}

Hospital Quirón Madrid. España.

Doctora en enfermería por la Universidad complutense de Madrid. Profesora del master propio de Comunicación y Salud de la Universidad Complutense. Colaboradora honorifica del Departamento de Teoría y Análisis de la comunicación de la Universidad Complutense. Miembro del grupo consolidado de investigación de la Universidad complutense "Creación y efectos Psicosociales del discurso audiovisual". Autora de diferentes artículos y capítulos de libro sobre comunicación y salud. Enfermera en la Uci de Neonatos del Hospital Quirón de Madrid.

victoria.cuesta.diaz@gmail.com

Orcid ID: https://orcid.org/0000-0003-1318-1173

\section{Luz Martínez Martínez}

Departamento de Teorías y Análisis de la Comunicación. Universidad Complutense de Madrid. España.

Doctora en Comunicación Audiovisual por la Universidad Complutense de Madrid. Especializada en el aprendizaje a través de los medios de comunicación y en comunicación y salud. Profesora asociada de la Universidad Rey Juan Carlos e Investigadora de la Cátedra de Comunicación y Salud del departamento de Comunicación, Audiovisual y Publicidad II de la Facultad de Ciencias de la Información (UCM). Profesora asociada en el Grado de Comunicación Audiovisual de la Universidad Rey Juan Carlos de Madrid. Ha publicado trabajos en revistas científicas y participado en numerosos congresos internacionales. Actualmente está integrada como investigadora en el laboratorio de Neuromarketing "NeurolabCenter" perteneciente al Departamento de Comunicación, Audiovisual y Publicidad II de la Facultad de Ciencias de la Información (UCM). Sus líneas de investigación se centran en: en el análisis de la creación y efectos psicosociales y culturales del discurso audiovisual en diferentes soportes, su aplicación en el edu-entretenimiento y el estudio de su eficacia a través de metodologías innovadoras, neuromarketing.

luzmar03@ucm.es

Índice H: 4

Orcid ID: http://orcid.org/0000-0001-8582-724X

Google Scholar: https://scholar.google.es/citations?user=AKGwpH8AAAAJ\&hl=es

\section{José Ignacio Niño González}

Departamento de Teorías y Análisis de la Comunicación. Universidad Complutense de Madrid. España.

Doctor en Publicidad y Relaciones Públicas por la Universidad Complutense de Madrid, y Máster en Administración de Empresas por el Instituto de Empresa de Madrid. Actualmente es Secretario de la Comisión Académica del Programa de Doctorado en Comunicación, Audiovisual, Publicidad y Relaciones Públicas que se imparte en la Facultad de Ciencias de la Información (UCM) y es Subdirector del laboratorio de Neuromarketing "NeurolabCenter" perteneciente al Departamento de Teorías y Análisis de la Comunicación de la Facultad de Ciencias de la Información (UCM). 
Miembro del Grupo de Investigación consolidado UCM "Creación y efectos psicosociales y culturales de la comunicación". Autor de múltiples artículos en revistas de investigación de impacto en español e inglés, capítulos de libro y libro en el ámbito del neuromarketing, comunicación y salud. En su trayectoria profesional destaca su etapa en agencias de publicidad como Tandem DDB y Saatchi \& Saatchi o proyectos como socio-fundador en el área de la comunicación y las nuevas tecnologías como Interacting o More Than Game.

jnino@ucm.es

Î́ndice H: 12

Orcid ID: http://orcid.org/0000-0001-6940-2399

Google Scholar: https://scholar.google.es/citations?user=yswmCT4AAAAJ\&hl=es 